\title{
Analysis of Suitable Electrode Positions for Writing Imagination
}

\author{
Talit Jumphoo ${ }^{1}$, Monthippa Uthansakul ${ }^{1}$ and Peerapong Uthansakul ${ }^{1+}$ \\ School of Telecommunication Engineering, Suranaree University of Technology, NakhonRatchasima, \\ Thailand 30000
}

\begin{abstract}
The human brain has many different functions and it is so complicated that each position on human head provides the different electroencephalography signal. It is interesting to find out the suitable electrode positions for a specific brain function. In this work, the writing imagination is on focus because it is curious which brain functions such as imagination, movement, memory, language and perception play the main role on the writing imagination. This paper analyzes to find the suitable electrode positions for recording the electroencephalography signals when we imagine to write either circle or straight line by using artificial neural networks. The results reveal that the suitable electrode positions are the frontal pair of F3 and F4channels. This outcome is very helpful for studying further on writing imagination in the future application.
\end{abstract}

Keywords: Frontal lobe, Electroencephalography, Cognitive, Artificial Neural Networks.

\section{Introduction}

In the past, Electroencephalography (EEG) signals recorded from medical equipment was expensive and complicated. But now the development of technology can create an affordable product such as the EEG headset providing a wireless connector such as Emotiv EPOC. It is inexpensive and easy to use so it is very popular in research [1], [2], [3]. Eomtiv EPOC has 14 electrodes (AF3, F7, F3, FC5, T7, P7, O1, O2, P8, T8, FC6, F4, F8, and AF4) [4]. Fig. 1 shows Emotiv's electrodes placement (Red) and the letters F, T, C, P and O stand for frontal, temporal, central, parietal, and occipital lobes, respectively. To choose a suitable electrode positions for each brain function allows a direct analysis and time saving. In [5], it is shown that the suitable electrode positions according to the brain function of emotion are T7 and T8. In another research studying the hand movement [6] [7], the suitable electrode positions are C3 and C4. In this study, the analysis of the suitable EEG electrodes for writing imagination without using arm muscle has been presented. This can be very useful for any future research that involves a writing imagination.

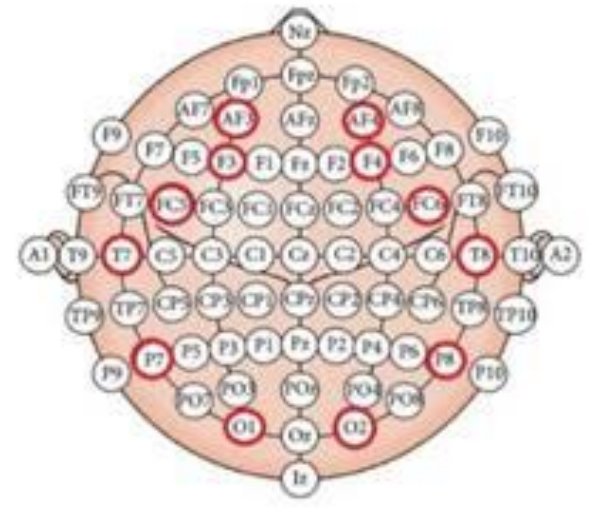

Fig. 1: International 10-20 system of electrode placement [5] and Emotiv's electrodes placement (Red).

\footnotetext{
+ Corresponding author. Tel.: +66850865588; fax: +6644224603.

E-mail address: uthansakul@ sut.ac.th
} 


\section{Methodology}

\subsection{Data Collection}

The data was collected by researcher. He wore an Emotiv EPOC headset and performed to look the animation of circle or straight line as shown in Fig.2. Then, the researcher imagined to write either circle or straight line. The EEG data was obtained by recording from the fourteen electrodes using raw EEG and the bandwidth: $0.2-43 \mathrm{~Hz}$, digital notch filters at $50 \mathrm{~Hz}$ and $60 \mathrm{~Hz}$ [4]. The duration of imagination is 8 seconds for each image. Fig. 3 shows the process of data collection which researcher imagines writing the circle in his imagination during the experiment. In this experiment, All EEG datasets were collected from imagining to an animation of circle for 100 datasets and straight line for 100 datasets. The measured EEG datasets will be used to train the artificial neural network learning later.
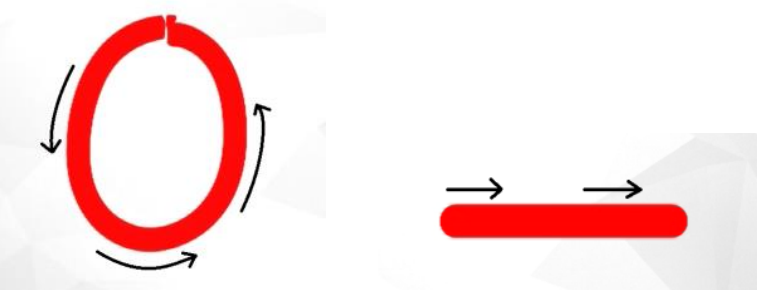

Fig. 2: The image of circle and straight line for collecting the EEG signals.

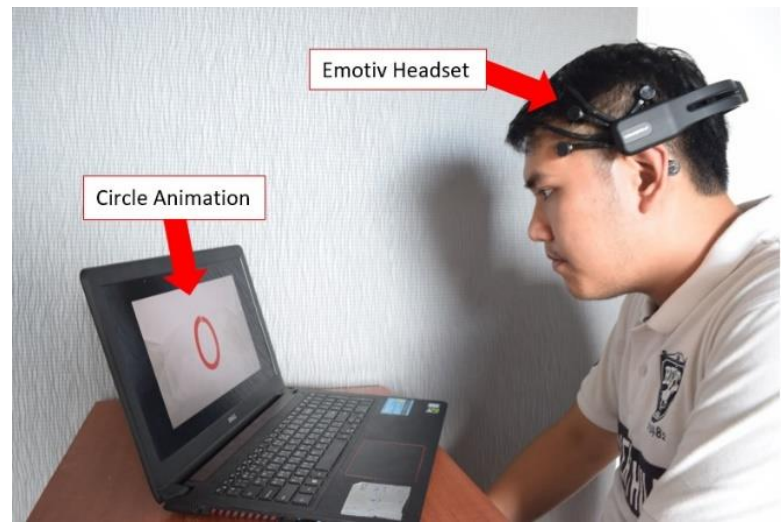

Fig. 3: Researcher imagines writing the circle in his imagination during the experiment.

\subsection{Learning Artificial Neural Network}

The EEG datasets are measured from 14 electrodes named as AF3, F7, F3, FC5, T7, P7, O1, O2, P8, T8, FC6, F4, F8 and AF4. The patterns of EEG signals are learnt by artificial neural network and then they will be compared between each other to find the suitable electrodes based on the correlation coefficients. The correlation coefficients provide the statistical relationships between two or more random variables and observed data values [8], [9] as shown in (1):

$$
r=\frac{\sum_{i=1}^{n}\left(x_{i}-\bar{x}\right)\left(y_{i}-\bar{y}\right)}{\sqrt{\sum_{i=1}^{n}\left(x_{i}-\bar{x}\right)^{2} \sum_{i=1}^{n}\left(y_{i}-\bar{y}\right)^{2}}}
$$

The EEG datasets were normalized by scaling between 0 and 1 as shown in (2) in order to reduce variability [5], [10]:

$$
\text { normalize }\left(X_{i}\right)=\frac{X_{i}-X_{\min }}{X_{\max }-X_{\min }}
$$

Fig. 4 shows the EEG signals of electrodes F3 and F4 which have the most correlation coefficients for recoding the writing imagination of circle(above) and straight line(below). Each electrode is used as input to the learning of individual neural networks. Fig. 5 shows a model of EEG data input of the artificial neural network and vectors of two components are defined as the output targets, Circle [0 1], Line [1 0]. 

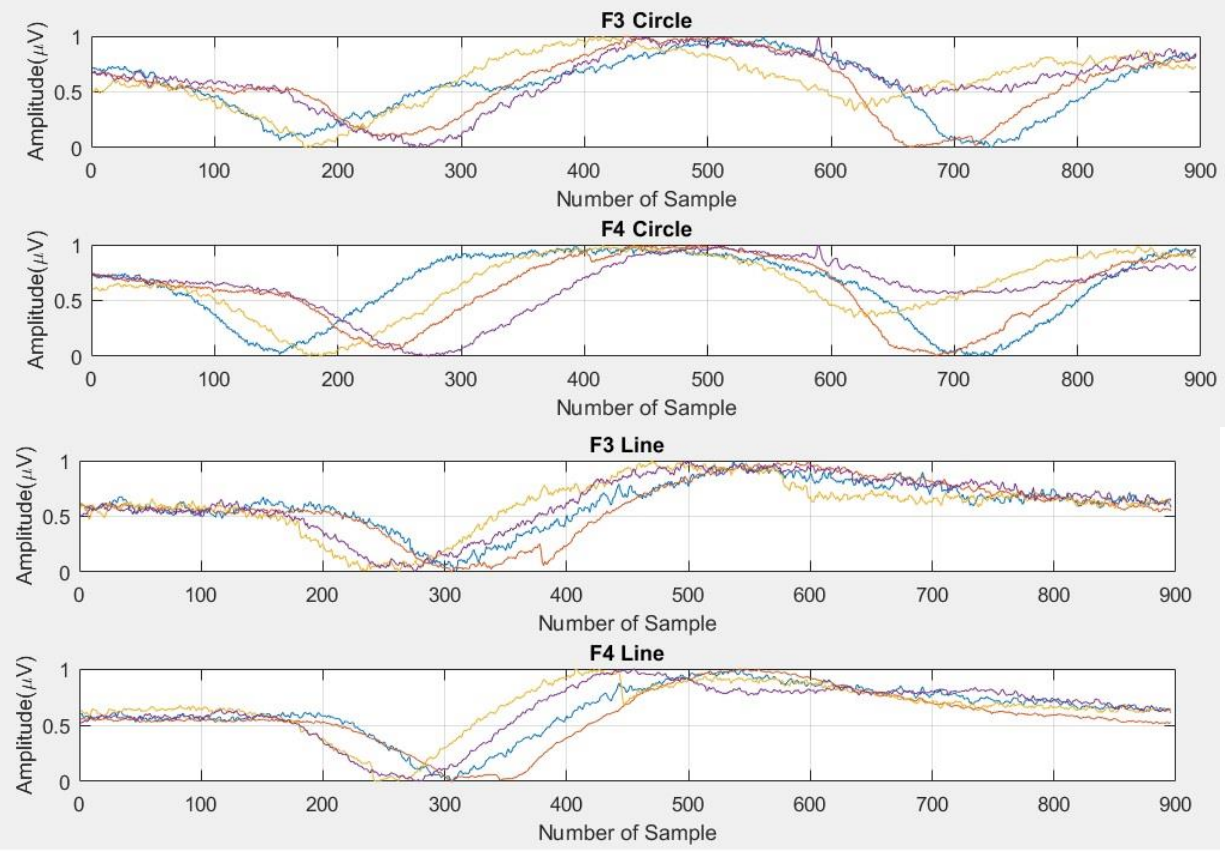

Fig.4: EEG signals of electrodes F3 and F4 which have the most correlation coefficients for recoding the writing imagination of circle (above) and straight line(below).

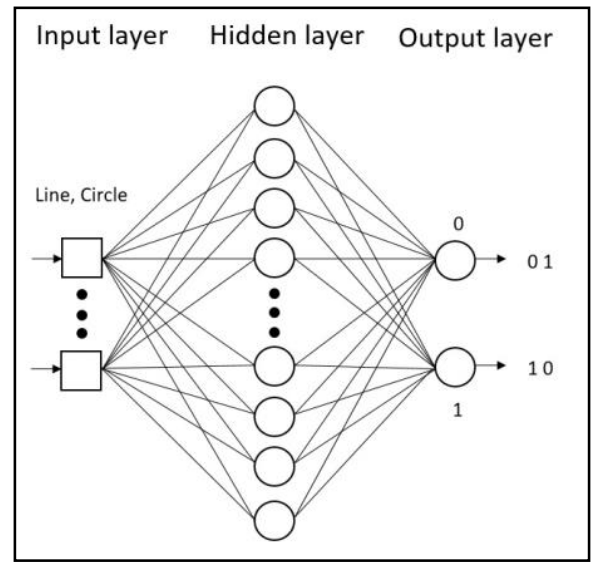

Fig.5: Input and output of artificial neural network.

\subsection{Testing Artificial Neural Network}

In this testing, the researchers who have no neuro disorder histories record their EEG signals in order to perform two tasks. For the first task, the researchers imagine to write a circle for 100 times and relax about 1 hour before starting the second task which is the recording of a straight line for 100 times. Then the recorded signals are used as data for testing artificial neural network.

\section{Experimental Results and Discussions}

Table 1 presents the classification accuracy of 14 electrodes (AF3, F7, F3, FC5, T7, P7, O1, O2, P8, T8, FC6, F4, F8 and AF4) when imagining to write a circle. The results reveal that the top-two highest average accuracies can be achieved at positions of F3 and F4 which the accuracies are $70 \%$ and $72.86 \%$, respectively. Table 2 presents the classification accuracy of 14 electrodes when researchers imagine writing a straight line. Interestingly, it can be found that the top-two highest average accuracies are at positions of F3 and F4 which the accuracies are $68.57 \%$ and $70 \%$, respectively. As seen from both tables, it can be seen that the electrode positions at AF3, F7, F3, F4, F8, and AF4 give the correct results more than 50\% when researchers imagine writing a circle and a straight line. From these experimental results, it can be explained that the electrode positions at AF3, F7, F3, F4, F8, and AF4 are placed on frontal lobe area which acts to control concentrating, thinking (such as ideation, imagination) and problem-solving. These experimental results get along with the 
area of frontal lobe which was described in Fig. 6 [11]. Note that the important problem in analysing EEG signals is that EEG signals can be generated when a muscle is shifting of the facial such as rise of brow or furrow of brow.

In [12], it was shown that the EEG signals were occurred by facial expressions in each electrode position. Remarkably, it was found that a pair of F3 and F4 channels provided the EEG signals with the minimum correlation to the facial expressions. The outcome of this work also presents the consistency with the research in [13] which a pair of F3 and F4 channels has an important role in the perception of information.

Table1: Classification accuracy of 14 electrodes when researchers imagine writing a circle.

\begin{tabular}{|c|c|c|c|c|c|c|c|c|c|c|c|c|c|c|}
\hline Circle & AF3 & F7 & F3 & FC5 & T7 & P7 & O1 & O2 & P8 & T8 & FC6 & F4 & F8 & AF4 \\
\hline $\begin{array}{c}\text { Circle(\%) } \\
{[01]}\end{array}$ & 65.71 & 68.57 & 70 & 40 & 47.14 & 42.86 & 64.29 & 62.86 & 58.57 & 44.29 & 48.57 & 72.86 & 61.43 & 67.14 \\
\hline $\begin{array}{c}\text { Line(\%) } \\
{[10]}\end{array}$ & 33.28 & 31.43 & 30 & 60 & 52.86 & 57.14 & 35.71 & 37.14 & 41.43 & 55.71 & 51.43 & 27.14 & 38.57 & 32.86 \\
\hline
\end{tabular}

Table2: Classification accuracy of 14 electrodes when researchers imagine writing a straight line.

\begin{tabular}{|c|c|c|c|c|c|c|c|c|c|c|c|c|c|c|}
\hline Line & AF3 & F7 & F3 & FC5 & T7 & P7 & O1 & $\mathrm{O} 2$ & P8 & T8 & FC6 & F4 & F8 & $\mathrm{AF} 4$ \\
\hline $\begin{array}{c}\text { Circle(\%) } \\
{\left[\begin{array}{ll}0 & 1\end{array}\right]}\end{array}$ & 32.86 & 34.29 & 31.43 & 37.14 & 38.57 & 41.43 & 68.57 & 65.71 & 57.14 & 37.14 & 38.57 & 30 & 37.14 & 34.29 \\
\hline $\begin{array}{c}\text { Line }(\%) \\
{\left[\begin{array}{ll}1 & 0\end{array}\right]}\end{array}$ & 67.14 & 65.71 & 68.57 & 62.86 & 61.43 & 58.57 & 31.43 & 33.28 & 42.86 & 62.86 & 61.43 & 70 & 62.86 & 65.71 \\
\hline
\end{tabular}

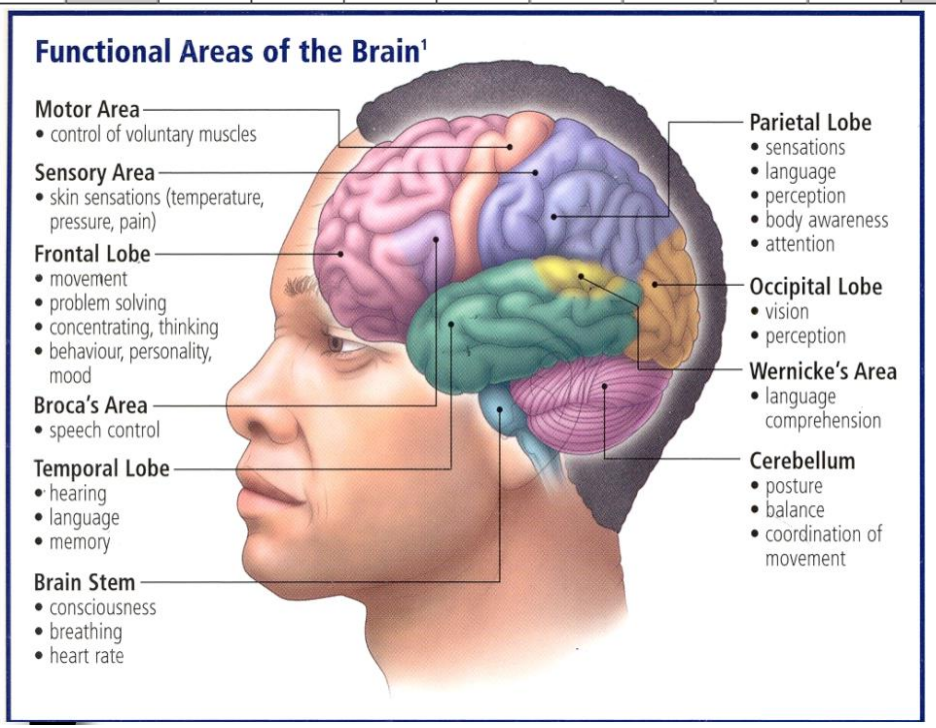

Fig. 6: Functional areas of the Brain [11].

\section{Conclusion}

Among all electrode positions on frontal area, the F3 and F4 positions are the suitable electrodes to record the EEG signals for studying writing imagination. These results are considered by using the correlation coefficients in the artificial neural networks. This conclusion is very helpful to investigate the EEG signals in the future because it can save more time and provide a direct analysis on this specific problem. For future work, the authors will apply this study to send some messages by interpreting the brainwave signals.

\section{Acknowledgement}

This work is financially supported from the Thailand Research Fund through the Royal Golden Jubilee Ph.D. Program (Grant No. PHD/0148/2557) 


\section{References}

[1] D. Prince, M. Edmonds, A. Sutter, M. Cusumano, W.Lu, and V. Asari, "Brain Machine Interface Using Emotiv EPOC To Control Robai Cyton Robotic Arm," Aerospace and Electronics Conference (NAECON) - 2015, 23792027, 15-19 June 2015.

[2] D. S. Benítez, S. Toscano and A. Silva, "On the use of the Emotiv EPOC neuroheadset as a low cost alternative for EEG signal acquisition ," Communications and Computing (COLCOM) - 2016, 27-29 April 2016

[3] Vourvopoulos, Athanasios, and Fotis Liarokapis. "Brain-controlled NXT Robot: Tele-operating a robot through brain electrical activity." Games and Virtual Worlds for Serious Applications (VS-GAMES), 2011 Third International Conference on. IEEE, 2011.

[4] Available at https://www.emotiv.com

[5] Jatupaiboon, Noppadon, Setha Pan-ngum, and Pasin Israsena. "Real-time EEG-based happiness detection system." The Scientific World Journal 2013 (2013).

[6] Upadhyay, R., et al. "Extraction and classification of Electroencephalogram signals." Computational Intelligence \& Computing Research (ICCIC), 2012 IEEE International Conference on. IEEE, 2012.

[7] Fadzal, CWNF Che Wan, W. Mansor, and L. Y. Khuan. "An analysis of EEG signal generated from grasping and writing." Computer Applications and Industrial Electronics (ICCAIE), 2011 IEEE International Conference on. IEEE, 2011.

[8] Üstündağ, Mehmet, et al. "Denoising of weak ECG signals by using wavelet analysis and fuzzy thresholding." Network Modeling Analysis in Health Informatics and Bioinformatics 1.4 (2012): 135-140.

[9] ÜSTÜNDAĞ, Mehmet, et al. "Performance comparison of wavelet thresholding techniques on weak ECG signal denoising." Przegląd Elektrotechniczny 89.5 (2013): 63-66.

[10] Lin, Yuan-Pin, et al. "Support vector machine for EEG signal classification during listening to emotional music." Multimedia Signal Processing, 2008 IEEE 10th Workshop on. IEEE, 2008.

[11] Available at https://avinashkm- neurosurgery.blogspot.com/2013/06/my-philosophy-of-neurosurgery.html

[12] L. Vokorokos, N. Ádám, B. Madoš, “ Non-Invasive Brain Imaging Technique for Playing Chess with BrainComputer Interface,” International Journal of Computer and Information Technology, ISSN: 2279 - 0764, Volume 03 - Issue 05, September 2014

[13] Knoll, Y. Wang, F. Chen, J. Xu, N. Ruiz, J. Epps and P. Zarjam, "Measuring cognitive workload with low-cost electroencephalograph,” In IFIP Conference on Human-Computer - (2011, September), Interaction (pp. 568-571). Springer Berlin Heidelberg. 